\title{
Progressive Point-Light-Based Global Illumination
}

Holger Dammertz, Alexander Keller, Hendrik P.A. Lensch

\section{Ulmer Informatik-Berichte}

Nr. 2009-09

Juli 2009 



\title{
Progressive Point-Light-Based Global Illumination
}

\author{
Holger Dammertz ${ }^{1}$ and Alexander Keller ${ }^{2}$ and Hendrik P. A. Lensch ${ }^{3}$ \\ 1 holger. dammertz@uni-ulm. de Ulm University, Germany \\ 2 alex@mental. com, mental images GmbH, Germany \\ 3 hendrik. lensch@uni-ulm. de Ulm University, Germany
}
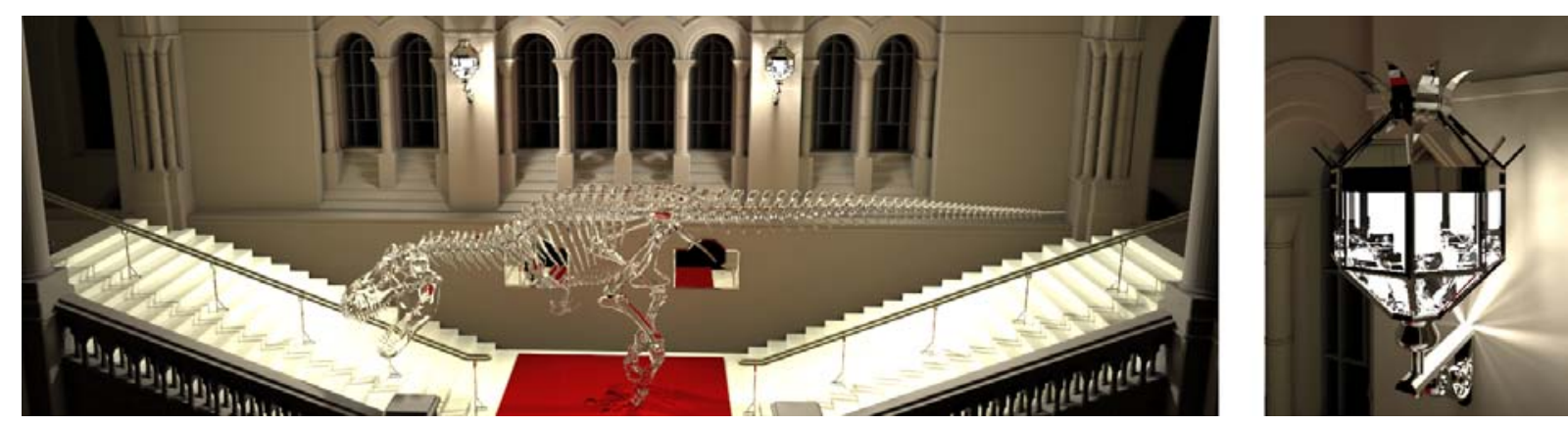

Figure 1: Our progressive rendering system is able to handle a wide range of complex light paths efficiently by combining three approaches. The shown scene contains diffuse and specular objects that are illuminated only by two lamps enclosed in glass. Our system not only handles the bright areas well but allows for smooth indirect illumination from this kind of light sources without the need for parameter tuning.

\begin{abstract}
We present a physically-based progressive global illumination system that is capable of simulating complex lighting situations robustly by efficiently using both light and eye paths. Specifically, we combine three distinct algorithms: point-light-based illumination which produces low-noise approximations for diffuse inter-reflections, specular gathering for glossy and singular effects, and a caustic histogram method for the remaining light paths. The combined system efficiently renders low-noise production quality images with indirect illumination from arbitrary light sources including inter-reflections from caustics and allows for simulating depth of field and dispersion effects. Our system computes progressive approximations by continuously refining the solution using a constant memory footprint without the need of pre-computations or optimizing parameters beforehand.
\end{abstract}

Categories and Subject Descriptors (according to ACM CCS): Computer Graphics [I.3.7]: Three-Dimensional Graphics and Realism: Color, shading, shadowing, and texture

\section{Introduction}

In this paper we present a progressive bidirectional physically-based rendering system that improves the perceived image quality in many situations.

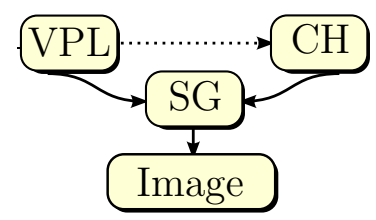

For this we combine three different techniques in a robust way. Each technique contributes a distinct part to the final image and together they are able to simulate for example caustics, specular surfaces, and depth of field effects.

The benefit of the combination is that the techniques can take advantage of each other by reusing information where appropriate and that the advantages of each technique are retained:

- virtual point light sources (VPL): smooth indirect illumination

- caustic histograms $(\mathrm{CH})$ : crisp, efficient caustics

- specular gathering (SG): robust handling of glossy and singular materials, 
The novel caustic histogram method collects photons in bins that are created for each of the progressively sampled eye paths. Although we include approximating techniques and thus accept a certain small bias in the solution, the progressive rendering algorithm consistently converges to a unique solution, especially in complex illumination situations.

We argue that a fully progressive system allows for much easier handling of the renderer by artists because the choice of parameters does not change the final image quality (they affect only rendering time). In addition, the proposed system decouples memory consumption and image quality (i.e. higher quality renderings do not require more memory at runtime). Compared to many previous approaches our system renders low-noise production quality images with indirect illumination from arbitrary light sources including interreflections from caustics and allows for simulating depth of field and dispersion effects.

\section{Related Work}

Physically-based rendering is a sub-problem of image synthesis, which aims to simulate light transport on a computer in a physically correct way. The problem has been studied in great detail [PH04, DBB06] and as it stands, ray tracing is the only method that allows to solve complex illumination problems consistently. Even though ray tracing performance has been improved greatly in the recent years [Shi06], only few of the achievements directly help Monte Carlo based global illumination algorithms [BWB08, DHK08]. Several hundreds of million paths need to be ray traced for a final image and thus Monte Carlo and quasi-Monte Carlo rendering algorithms have a very high computational demand and long rendering times. Unbiased Monte Carlo-based rendering systems are progressive by nature and thus have the advantage of robustly handling even complex situations with the only limitation being the rendering time. The disadvantage is that even in simple situations unbiased Monte Carlo algorithms have a high variance perceivable as noise in the image.

Kajiya [Kaj86] introduced Monte Carlo path tracing algorithms to computer graphics. Since then, a lot of research headed to improve the efficiency of the basic algorithm. The family of bidirectional path tracing algorithms using multiple importance sampling [LW93, VG94] and the Metropolis light transport algorithm [VG97] belong to the most powerful algorithms to date. While these algorithms are unbiased and can deal with complex lighting situations, they suffer from variance, which becomes visible as noise in images. Our system approaches the global illumination from both light and eye paths using a Monte Carlo technique, however, it reduces variance through the use of correlated point light sources for diffuse inter-reflections.

The Instant Radiosity algorithm [Kel97] uses point light sources and graphics hardware to quickly compute a global illumination solution for diffuse scenes. Since then, pointlight-based global illumination has proven to be a useful and efficient way to approximate diffuse inter-reflection in real time systems [BWS03] and preview systems [HPB07]. In [SIMP07] an extension to the Instant Radiosity algorithm is presented that uses Metropolis sampling to increase efficiency. The use of VPLs is at the core of our rendering system, however, we extend it to simulate non-diffuse light paths as well.

Photon mapping [Jen96] has been introduced as a solution to the problem that there exist paths that cannot be efficiently sampled by any unbiased technique. With the recent improvements [HOJ08], many shortcomings of the original method have been removed. In our approach we remove the remaining memory bound on the number of eye path vertices that need to be stored, which for example allows one to simulate anti-aliased depth of field with any sampling rate required.

The Lightcuts rendering framework [WFA* 05 , WABG06] is a powerful approach to the many-lights problem, which is entirely based on point-light sources. Due to the ability to deal with an enormous number of point light sources, glossy effects can be handled, however, the system cannot simulate caustics and is not progressive. As our approach is progressive there is no limitation on the number of light sources and even the simulation of caustics is integrated.

\section{System Overview}

Our physically-based rendering system is designed to produce production quality images of 3D scenes with global illumination in complex situations without the need for extensive parameter tweaking. We therefore assume physically plausible input: for example light sources are modeled as geometry that is part of the scene, glass objects always have a thickness, and surface shaders need to be energy conserving. Progressiveness enables the user to continue image computation until a satisfactory result is achieved. There is neither the need to restart computations nor to discard intermediate results that allow for previews of an illumination situation after a short amount of time.

The goal of our system is to provide a fast and smoother global illumination solution as compared to approaches using multiple importance sampling [VG95, Vea97, VG97] without sacrificing too much quality and flexibility. We achieve this goal by combining three techniques, each of which simulates a disjoint subset of path space in such a way that the techniques benefit from each other. For ease of reading, we apply Heckbert's [Hec90] notation to identify path subspaces: $E$ is the eye or camera, $D$ denotes a diffuse bounce (in Section 4 we define what diffuse means for our rendering system), $S$ is a non-diffuse (specular) bounce, and $L$ represents the light sources. 


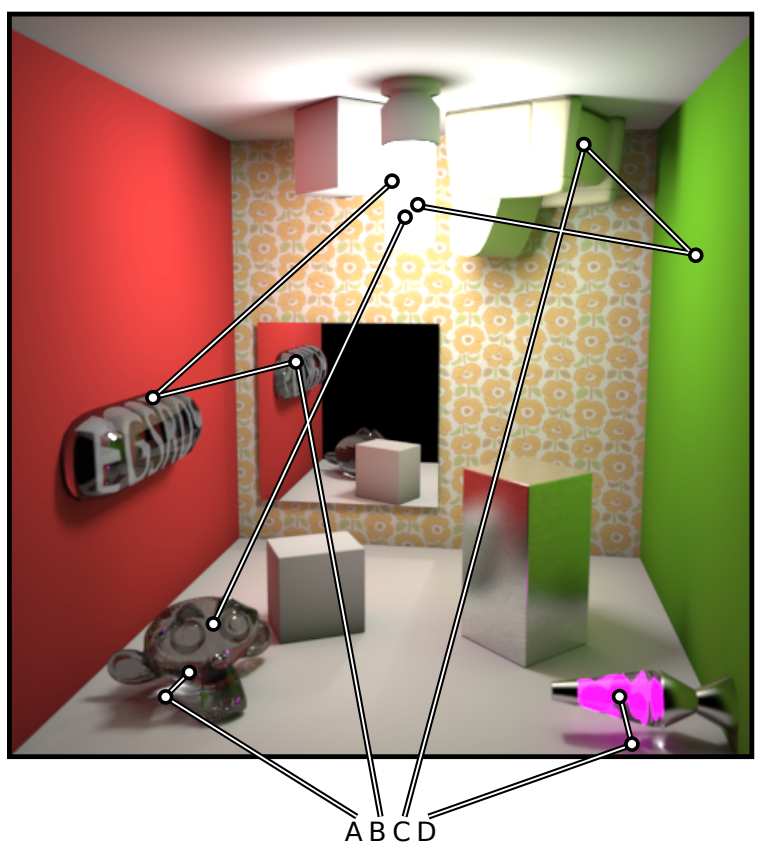

Figure 2: Illustration of selected light paths that we are able to simulate robustly and consistently: 'A' is a classic caustic path (EDSSL), 'B' shows the mirror image of a diffuse $o b$ ject behind a glass object (ESSDSL), 'C' is diffuse indirect illumination from the green wall to the sofa $(E D D L)$, and 'D' shows a complex shaped light source (lava lamp) behind glass illuminating the scene (EDSL). Also note the caustic generated by the monkey head in the mirror (ESDSSL).

Figure 2 illustrates some of the light transport paths we are able to simulate. The techniques and the simulated light paths in our system are:

$E D^{+} L$ : is handled by the point-light-based algorithm (Section 5.1) (also handles $E D D\{D \mid S\}^{*} L$ )

$E D S^{+} L$ : is created by the caustic histogram method (Section 5.3)

$E S^{+} D L$ : is handled by specular gathering (Section 5.2) and uses the results of the previous two methods

$E S^{*} L:$ is directly evaluated as in a path tracer [DBB06]

$E D S^{+} D^{+} L$ : caustics from indirect illumination are handled by the combination.

Looking at the path notation it can be seen that all these contributions are distinct and two methods can never generate a contribution for the same path. The final solution is simply obtained by summation. However, our system is biased, as we make several approximations that are described and discussed in the following.

Figure 3 shows the steps our rendering system performs in each rendering pass. A rendering pass is one iteration of computation that is finally added to the accumulation buffer containing the progressively refined result image. In the following sections each of the steps is explained in detail.
Store Eye Path Vertices on Diffuse Surfaces and compute Ray Differentials

Select Visibility Representants from Eye Path Vertices (VPLs rejection)

Generate Light Source Samples (primary VPLs)

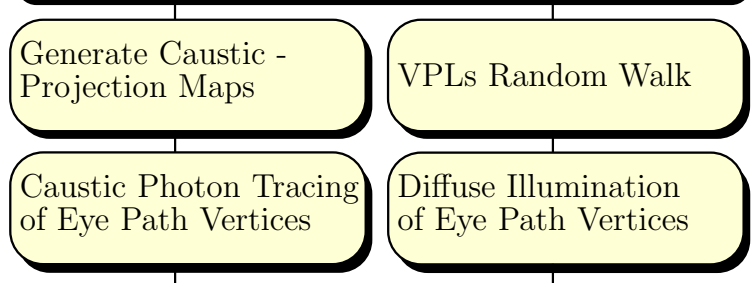

Filtered Accumulation Buffer

Figure 3: This figure describes the order of the basic operations our rendering system performs each rendering pass.

\subsection{Eye Path Generation}

The first step of our rendering system is the generation of eye path vertices. This is illustrated in Figure 4 (a). The eye paths are created by a random walk starting from the camera and are terminated via Russian roulette or when they hit a diffuse surface. The vertices are stored only on diffuse surfaces. These points are similar to the gather points in the Lightcuts [WABG06] algorithm (but we store only the end points of a path) or the hitpoints stored in the progressive photon map algorithm [HOJ08]. The main difference is that we create a new set of eye path vertices each rendering pass instead of keeping them for the whole image generation process. This allows for progressive refinement of spatial anti-aliasing and complex specular effects. In the standard setting one eye path is started per pixel for each rendering pass. When such a path hits a light source (either directly or via an arbitrary number of specular bounces: $E S^{*} L$ ) the contribution is directly added into the accumulation buffer but the path is not necessarily terminated as the surface of the light source might also reflect light. Using the generated eye path vertices we will gather diffuse illumination (Section 5.1), specular contributions (Section 5.2) and caustics (Section 5.3).

In order to estimate the bin size in the caustic histogram (Section 5.3) we also need a size associated with each eye path vertex. For this, we trace ray differentials [Ige99] starting with half the pixel size. This provides a good estimate of projected pixel footprint even after specular bounces.

\section{Material Model}

While general production renderers for the movie industry can use arbitrary complex programs to define surface 
color and reflection behavior [DH05, Kes08], physicallybased systems are more restricted in their choice of reflection functions [PH04]. Many practical algorithms exploit the fact that BRDFs are not only energy-conserving, but also provide an efficient sampling function.

A wealth of surface reflection models has been developed in computer graphics that provide most of the required functionality [Bli77, War92, AS00]. These reflection models allow one to describe a wide variety of basic surface types. For more complex surface behavior usually several reflection models are combined to a single layered material [PH04].

Systems using multiple importance sampling [PH04] require the BRDF model to satisfy the Helmholtz reciprocity condition in order to obtain consistent results. Since we partitioned path space and use only one technique on each partition, this condition no longer needs to be fulfilled, allowing one to use more convenient approaches like e.g. the halfway vector disc model $\left[\mathrm{EBJ}^{*} 06\right]$, while still enjoying convergence to a unique solution.

In order to determine the partition of the path space, we need to decide when a reflection is almost diffuse. In principle our system can use any physically-based layered BRDF model for which a parameter $\kappa$ can be assigned to each layer expressing how diffuse it is. This parameter is assumed to be normalized such that for $\kappa=0$ the layer is perfect Lambertian and for $\kappa=1$ the surface is a perfect mirror. During rendering each sample evaluates only one material layer that is selected by random sampling. We use the threshold of $\kappa=0.2$ for classifying a material layer as diffuse or specular.

For example, in all renderings we use the Blinn-Phong model [Bli77] with Phong-exponent $k=1024 \kappa+1$. Layered materials such as simple metal or glass (one reflecting, one transmitting layer) and more complex materials like coated plastic can be constructed easily using the Fresnel term or one of the approximations [Sch94] for weighted sampling of material layers. For transmissive materials the direction is selected according to Snell's law but the width of the lobes is controlled as before, as this allows for diffuse and imperfect glass.

\section{Bidirectional Global Illumination}

Based on the material properties we partition the path space. In the following we describe how the different subsets are sampled.

\subsection{Point-Light-Based Diffuse Illumination}

We approximate the diffuse illumination by virtual point light sources (VPL) similar to the Instant Radiosity algorithm [Ke197]. In order to be consistent with existing literature we use the term VPL (Virtual Point Lightsource) for a light path vertex even though we prefer the term DIAL (Diffuse Illumination-Approximating Light) in the context of our rendering system. Note that the notion of diffuse illumination not only includes perfectly Lambertian surfaces, but also slightly glossy surfaces as specified in Section 4 .

The first step in the Instant Radiosity algorithm generates samples on the light sources. As our scenes contain arbitrarily shaped light sources modeled as triangles, we first compute a PDF according to the area and intensity of each emitting triangle. This PDF is created once and can be reused for all subsequent rendering passes. Using three random numbers, a position on the light sources (stored as primary VPL) is sampled. Then a random walk terminated by Russian roulette is performed in the manner of a particle tracer, where on each diffuse surface another VPL is stored. Unlike eye paths, the light paths are not terminated on the first diffuse hit. This is illustrated in Figure 4 (b). These VPLs represent a point-wise approximation of the diffuse interreflection. In contrast to [Ke197], the contribution of each VPL is then accumulated by sampling the direct visibility of the VPLs to each pixel via the stored eye path vertices, i.e. possibly incorporating even multiple specular bounces (see next section). Figure 4 (c) illustrates this. This accumulation is inherently progressive as each series of VPLs, generated by one random walk, is independent of the other random walks and the final image is independent of the number of VPLs created per rendering pass.

Stochastic Culling of VPLs. As we want to be able to efficiently handle complex scenes, we use an additional optimization to discard VPLs that are likely to have no contribution. For this purpose we choose a stratified subset of 256 of the eye path vertices and check the visibility from these locations prior to VPL storage. This can be either done based on an acceptance probability to not introduce an additional bias, or fully deterministic. To guarantee a good coverage and a changing subset for each rendering pass we use the Halton sequence over the image plane for the selection [KW00].

\subsection{Glossy and Singular Effects}

Glossy and singular effects need to be simulated when the BRDF is not sufficiently diffuse (see Section 4). The idea of specular gathering is to sample the BRDF according to its probability density function by extending the eye path. This extension of the eye path is repeated until either it is terminated by Russian roulette or the path hits a diffuse surface. At this point the illumination is computed by using the point light sources and the caustic histogram (see next section). So, per rendering pass we gather the illumination for specular eye paths by using the current global illumination approximation given by the two other techniques. As this approximation is recomputed in each pass, the specular part becomes progressively refined, too. 


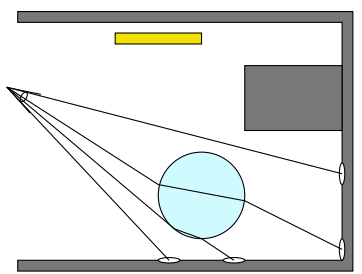

(a)

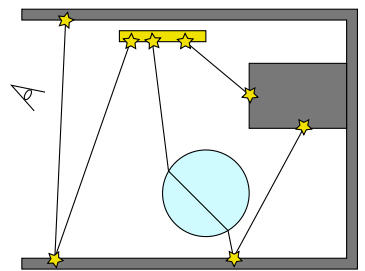

(b)

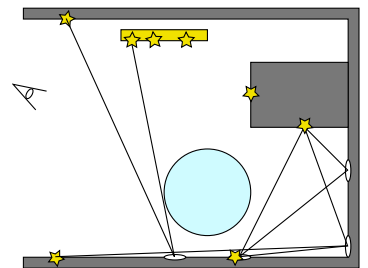

(c)

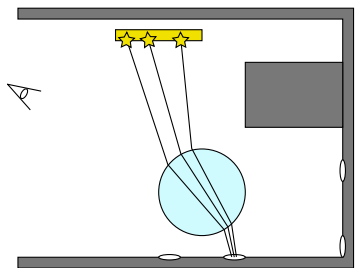

(d)

Figure 4: $(a)$ : Generation of the eye path vertices by performing a random walk into the scene starting from the eye. (b): Generation of the VPLs by a random walk starting from light sources. (c): Evaluation of the VPLs to compute an approximation of the diffuse illumination at the eye path vertices. $(d)$ : Accumulation of caustic photons into eye path vertices.

\subsection{Caustic Histogram Method}

The final technique of our rendering system is the histogram method that provides the caustic paths. From each primary VPL we trace photon random walks into the scene. If they do not hit a specular material they are discarded, otherwise they are continued until a diffuse material is found (or they are terminated by Russian roulette). There, they are accumulated into bins associated with each eye path vertex via a $k$ d-tree. The bin size varies per vertex (see Section 3). This procedure is illustrated in Figure 4 (d) for a glass sphere. In our implementation we trace photons until we have accumulated a given number (100000 in all images) for each rendering pass. Using the previously computed VPL as emitting light sources has the advantage of not requiring a new set of light source samples.

This method is conceptually similar to the progressive photon mapping algorithm [HOJ08]. However, first, we only use the caustic histogram for the $L S^{+} D S^{*} E$ paths, and second, constant radius per eye path vertex is used for the bins based on the projected pixel size, which speeds up the photon collection. Furthermore, we recreate the bins in each rendering pass and thus in addition are able to support progressive per pixel anti-aliasing, glossy surfaces, depth of field, and motion blur. The fixed radius selection may of course introduce the usual photon mapping artifacts and introduces a bias, but as the (projected) bin size is not larger than half the pixel size the error is not larger than the error introduced by image space filters [SW00].

Projection Maps. Even for arbitrarily complex light sources only relatively few VPLs are generated per rendering pass. As we emit caustic photons from these VPLs we can make efficient use of a projection map per VPL as opposed to previous approaches of using projection maps with photon emission [Jen01].

Projection maps are a pre-computation to direct caustic photons only in areas where glossy/specular objects are. This increases the efficiency in simple scenes (with few glossy objects) significantly without slowing down complex scenes.

We create the projection map as a conservative projection

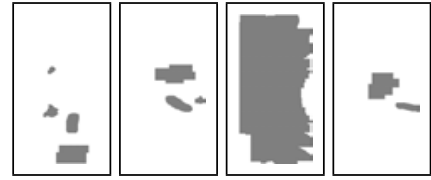

Figure 5: Illustration of four of the projection maps of the scene shown in Figure 10. The third map is from a VPL inside the lava lamp.

of all triangles classified as specular onto the hemisphere of each VPL and then use correctly weighted sampling to reject photon rays that would surely hit only diffuse surfaces. In order to efficiently splat the spherical triangles we approximate them by the bounding box of the projected vertices. Figure 5 shows some projection maps created in the Box scene. We used a resolution of $128 \times 256$ for each projection map. During rendering the generation of projection maps was between $2 \%$ and $5 \%$ of the total time spent on the caustic histogram method. The speed-up achieved is linear to the coverage of the hemisphere, saving $85.2 \%$ of the photon rays in the Box scene, corresponding to $65.3 \%$ of the time required to compute the caustics and $4.6 \%$ of the total render time.

Implementation Details. In practical applications the number of eye path vertices per rendering pass is larger than our chosen number of photons per pass. Since our accumulation of photons into vertices is redone every pass we can reverse the order and store the photons instead of the eye path vertices as in the original photon map algorithm [Jen96]. This does not change the result of the computation but may be more memory efficient for high resolution images.

\section{Lazy Spectral Rendering}

For efficiency reasons our system is based on RGB rendering. Yet it is possible to consistently integrate an approximation for dispersion effects into our rendering system by lazy spectral rendering. This works by using the normal RGB transport until a ray hits a dispersive surface (e.g. glass). At this point the ray is assigned a randomly sampled wavelength from the original RGB color. In addition, we further assign a novel RGB color corresponding to the sampled wavelength. 


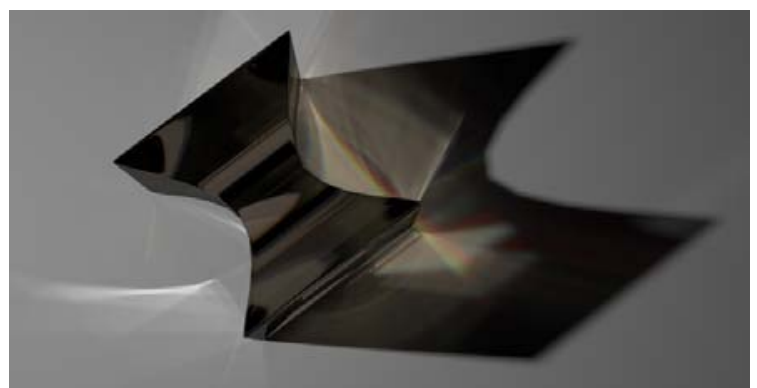

Figure 6: On demand spectral rendering allows to integrate dispersion into an $R G B$ based render.

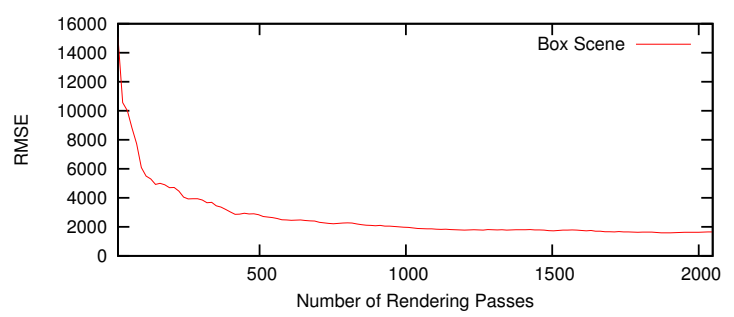

Figure 7: This graph shows the errors of the images (computed at $1024 \times 1024)$ shown in Figure 10 from 16 to 2048 rendering passes in steps of 16 compared to a reference image with 11456 rendering passes. The steep descent at the beginning is because the indirect illumination is quickly approximated by the point light sources.

Once a ray has an assigned wavelength it will keep it until the ray terminates.

The wavelength assignment is done for all rays generated in the system: eye-paths, photon-paths and the random walks to generate the VPLs. This allows for the usual color caustics (photon paths), the chromatic aberration seen through refractive objects (eye-paths) and correct indirect illumination from colored caustics (VPLs). Figure 6 shows a rendering with a dispersive glass object.

\section{Results}

In this section we discuss the results of our rendering system. All images in this paper were computed using the same default parameter settings of 16 VPL paths, 1 eye path per pixel, and 100000 caustic paths per rendering pass.

Figure 11 shows the statistics for the different scenes used to present different aspects of our system. Figure 10 shows the image generation process with the inverse difference image scaled by 8 compared to a reference image with 11456 render passes. In Figure 7 you can find the associated graph showing the mean square error.

Diffuse Illumination. The diffuse illumination (direct and indirect) is approximated by the use of VPLs and thus very
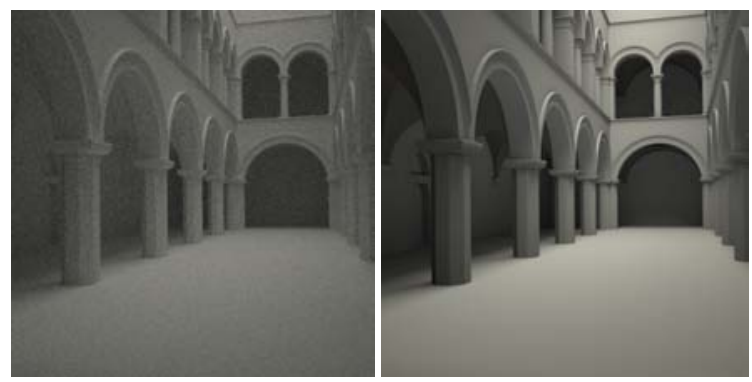

Figure 8: Equal time comparison (10 minutes on a single thread Core $22.33 \mathrm{GHz}$ ) of a BDPT (left) and the point light based renderer (right) in a simple diffuse scene. The noise in the BDPT solution is especially noticeable in the dark areas where none of the details are yet visible while in the other image the door and ceiling is clearly visible on the left side.

robust. This method was already discussed in many publications and is known to produce good results with a sufficient number of point light sources. As our system is progressive, the only limiting factor for the number of point light sources is rendering time. Figure 8 shows an equal time comparison of a bidirectional path tracer (BDPT) and point light based global illumination. The difference is most noticeable in the darker areas where the detail is still obstructed by noise in the BDPT while the VPLs approximation already shows each feature clearly. This robustness of indirect illumination in darker areas can also be seen in Figure 1 where the far left and right sides are only illuminated by indirect light. Note that the use of point lights for diffuse illumination also allows for efficient handling of diffuse transparent objects like curtains by creating VPLs also on the exit points (two sided) during the random walk.

Glossy Effects. Figure 9 shows a simple single layered material where $\kappa$ is varied from 0 to 0.9 . The first three spheres in the last row have a $\kappa \in(0,0.1,0.199)$ and are thus illuminated by VPLs directly (the specular threshold is $\kappa \geq 0.2$ ). The remaining spheres are illuminated by specular gathering, i.e. by extending the eye paths. Note, how these two techniques generate a consistent transition.

The top right image in Figure 11 shows a scene with many glossy objects and high variance situations where glossy objects are reflected in other glossy objects. In addition, the scene features depth of field. The illumination comes from a sky model only through the two small windows. The image was computed at a resolution of $960 \times 600$ with 52224 rendering passes for a good visual quality of the high variance areas (there is still some noticeable noise in the back of the room). Using this many gather points with progressive photon mapping would be infeasible: assuming 76 bytes per gather point as in the original paper [HOJ08] 2.1 terabyte of data would be generated.

Caustics and other $S D S$ paths. Caustics and $S D S$ paths are efficiently treated by the caustic histogram method us- 


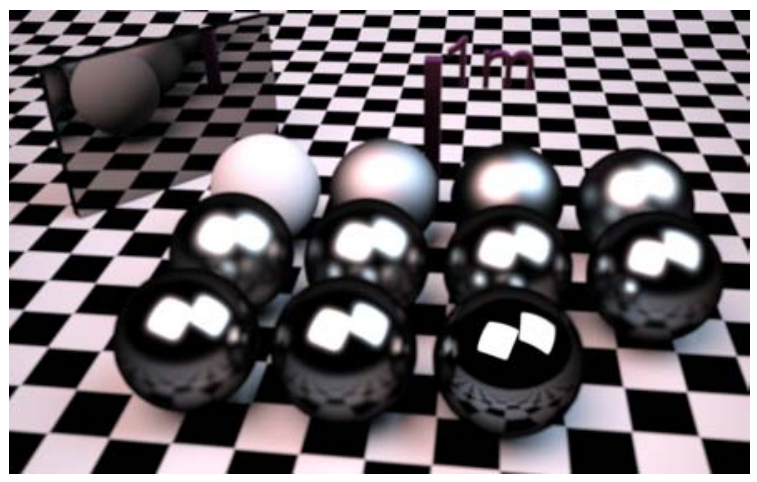

Figure 9: This image shows the smoothness of changing from VPLs based illumination for diffuse illumination $(\kappa<$ 2 , Section 5.1) to specular surfaces $(\kappa \geq 2$, Section 5.2). Due to the progressive rendering both methods converge to a final solution where no difference is noticeable without the need to set the correct number of samples or number of point light sources.

ing projection maps (Figure 1 and Figure 2). The $S D S$ paths are especially difficult in BDPT-based renderers and take a long time to converge even with Metropolis sampling. Without them the ground below the feet of the glass dinosaur in Figure 1 would be black. Section 5.3 further demonstrates how spectral effects can be integrated in our rendering system. The effect of spectral rendering is most noticeable in caustic areas, however, the presented method integrates well with the other proposed parts of the rendering system.

Depth of Field. Since we create a new set of eye path vertices in each rendering pass we can easily simulate e.g. a thin lens camera that renders depth of field effects. As our system is progressive we do not need to adjust any of the parameters for the illumination computation and, in many cases, the number of rendering passes needed for the illumination to converge is sufficient to robustly estimate depth of field effects (Figure 11).

\section{Limitations}

Indirect caustics $\left(E D S^{+} D^{+} L\right)$ are created from non-primary VPLs. This works well when diffuse surfaces are brightly lit, but in many scenes this increases the variance too drastically for the final visible effect. So we chose to make them artist-controllable instead of enabling them by default. Furthermore, the depth of indirect caustics can be selected prior to rendering.

The bias of bounding the geometric term in the Instant Radiosity method can be removed by the method proposed in [KK04]. The clamped contribution of point lights is compensated by gathering the missed illumination through additional rays. This comes at the cost of increasing the variance, especially in corners for an effect that is only seen in few scenes. We again made this an artist-controllable option.
While our proposed rendering system is progressive by design, it is not yet adaptive in any way (except that in highly glossy scenes automatically fewer VPLs are stored). Especially in diffuse areas the resulting image quickly converges to an acceptable solution while in areas of SDS paths there is still noticeable variance. By intuition one would like to sample only in areas of high variance. But this would remove some of the advantage of a real progressive renderer as it cannot be guaranteed that the undersampled region is no area of higher variance (for example light through a key hole) that was not yet sampled. Of course knowledge about a given scene allows an artist to increase convergence speed by tuning the number of point light sources or caustic photons per rendering pass accordingly.

\section{Conclusion}

We showed how a combination of three distinct techniques based on point light sources allows us to compute a global illumination solution including caustics, specular surfaces, depth of field, and dispersion effects in a single unified rendering system. Each of the techniques - VPLs, specular gathering and the novel caustic histograms - efficiently works with the other two in combination. The progressive nature of our system allows the artist to start a rendering and be sure that it will converge to a unique solution independent of the parameter choice. At least in theory our system would produce the same image even if just a single VPL path or a single photon path would be generated per rendering pass. Additionally, our system uses only a constant amount of memory during rendering independent of the final image quality.

Future Work. Subsurface scattering can be integrated in our system by allowing eye path vertices inside solid objects and tracing the photons also through these objects. Additionally, participating media using the VPLs can be integrated [RSK06]. Adding motion blur requires to sample just another dimension in our progressive renderer [CFLB06]. A desirable feature for the whole rendering system would be to automatically balance the three parameters (number of VPLs, number of specular gathering rays, and the number of photons for the histogram) for optimal convergence speed with respect to a given scene.

\section{Acknowledgments}

The first author would like to thank mental images $\mathrm{GmbH}$ for support and funding of this research. The first author would also like to thank Johannes Hanika and Matthias Raab for endless discussions about physically based rendering. 

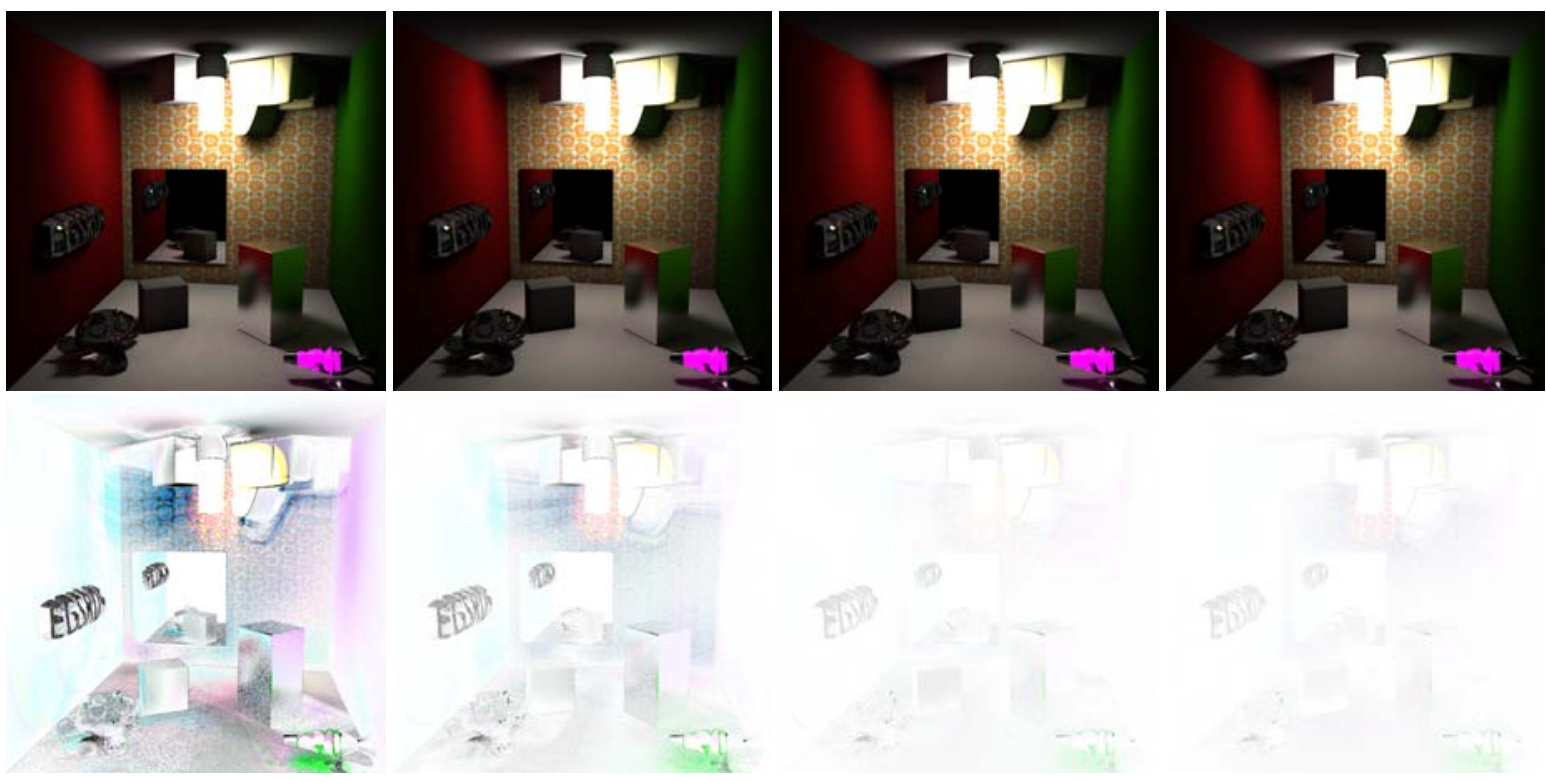

Figure 10: Evolution of the error with increasing number of rendering passes compared to a reference image with 11456 rendering passes (resolution: $1024 \times 1024)$. The top row shows the results after 16,128,512 and 2048 rendering passes. The bottom row shows the inverse difference image scaled by 8. Figure 7 shows the graph of the errors for the full image series.

\section{References}

[AS00] Ashikhmin M., Shirley P.: An anisotropic Phong BRDF model. Journal of Graphics Tools 5, 2 (2000), 25-32.

[Bli77] BLINN J.: Models of light reflection for computer synthesized pictures. SIGGRAPH Comput. Graph. (1977), 192-198.

[BWB08] Boulos S., Wald I., Benthin C.: Adaptive ray packet reordering. IEEE Symposium on Interactive Ray Tracing (Aug. 2008), 131-138.

[BWS03] Benthin C., Wald I., Slusallek P.: A Scalable Approach to Interactive Global Illumination. Computer Graphics Forum 22, 3 (2003), 621-630. (Proceedings of Eurographics).

[CFLB06] Christensen P., Fong J., Laur D., Batali D.: Ray tracing for the movie 'Cars'. In Proc. 2006 IEEE Symposium on Interactive Ray Tracing (2006), pp. 73-78.

[DBB06] Dutré P., BAla K., BeKaert P.: Advanced Global Illumination. AK Peters, Ltd., 2006.

[DH05] Driemeyer T., Herken R.: Programming Mental Ray (mental ray®Handbooks). Springer-Verlag New York, Inc., 2005.

[DHK08] Dammertz H., HaniKa J., Keller A.: Shallow bounding volume hierarchies for fast SIMD ray tracing of incoherent rays. In Computer Graphics Forum (Proc. 19th Eurographics Symposium on Rendering) (2008), pp. 1225-1234.

[EBJ*06] Edwards D., Boulos S., Johnson J., Shirley P., ASHIKHMIN M., STARK M., WyMAN C.: The halfway vector disk for BRDF modeling. ACM Transactions on Graphics 25, 1 (2006), 1-18.

[Hec90] HECKBERT P.: Adaptive radiosity textures for bidirectional ray tracing. In SIGGRAPH '90: Proceedings of the 17th annual conference on Computer graphics and interactive techniques (1990), pp. 145-154.

[HOJ08] Hachisuka T., Ogaki S., Jensen H.: Progressive photon mapping. In SIGGRAPH Asia '08: ACM SIGGRAPH Asia 2008 papers (2008), pp. 1-8.

[HPB07] HaŠAn M., Pellacini F., Bala K.: Matrix rowcolumn sampling for the many-light problem. ACM Trans. Graph. 26, 3 (2007), 26.

[Ige99] IGEHY H.: Tracing ray differentials. In SIGGRAPH '99. Proceedings of the 26th annual conference on Computer graphics and interactive techniques (1999), pp. 179-186.

[Jen96] JENSEN H.: Global illumination using photon maps. In Rendering Techniques '96 (Proc. of the Seventh Eurographics Workshop on Rendering) (1996), pp. 21-30.

[Jen01] JENSEN H.: Realistic image synthesis using photon mapping. A. K. Peters, Ltd., 2001.

[Kaj86] KAJIYA J.: The rendering equation. SIGGRAPH Comput. Graph. 20, 4 (1986), 143-150.

[Ke197] Keller A.: Instant radiosity. ACM Transactions on Graphics (Proc. SIGGRAPH 1997) (1997), 49-56.

[Kes08] Kesson M.: Pixar's renderman. In SIGGRAPH Asia '08: ACM SIGGRAPH ASIA 2008 courses (2008), pp. 1-138.

[KK04] Kollig T., Keller A.: Illumination in the presence of weak singularities. Monte Carlo and Quasi-Monte Carlo Methods 2004 (2004), 245-257.

[KW00] Keller A., WALD I.: Efficient importance sampling techniques for the photon map. In Proceedings of the Vision Modeling and Visualization Conference (2000), pp. 271-279.

[LW93] Lafortune E. P., Willems Y. D.: Bi-directional path 


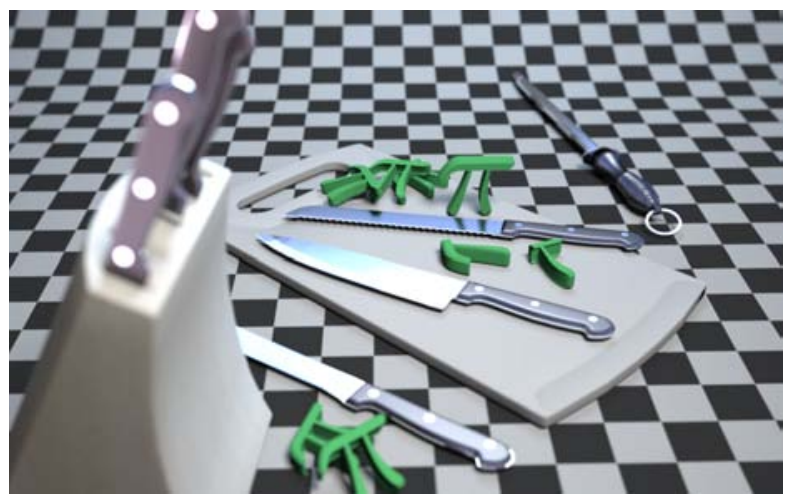

Knives: thin lens depth of field simulation

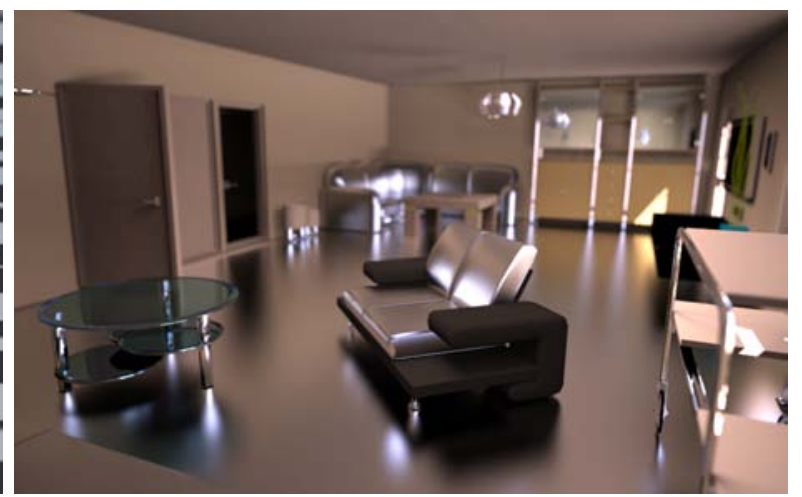

Interior: many glossy materials and depth of field

\begin{tabular}{|c|r|r|r|r|r|r|r|}
\hline Scene & Res. & Polygons & Avg. VPL & \% Diffuse & \% Eye Paths & \% Caustic & Time per Pass \\
\hline \hline Box & $1024 \times 1024$ & 49344 & 35.7 & $76.5 \%$ & $5.7 \%$ & $13.4 \%$ & $18.7 \mathrm{~s}$ \\
\hline Museum & $1200 \times 520$ & 331843 & 7.9 & $35.6 \%$ & $21.7 \%$ & $37.6 .8 \%$ & $4.6 \mathrm{~s}$ \\
\hline Interior & $960 \times 600$ & 909441 & 3.8 & $43.4 \%$ & $35.3 \%$ & $9.3 \%$ & $3.9 \mathrm{~s}$ \\
\hline Knives & $960 \times 600$ & 95472 & 22.1 & $52.0 \%$ & $6.2 \%$ & $39.2 \%$ & $11.9 \mathrm{~s}$ \\
\hline
\end{tabular}

Figure 11: The statistics show the relative time spent on the three techniques. The remaining time to $100 \%$ is due to management overhead. The projection map generation was between $2 \%$ and $5 \%$ of the total caustic time. The last column shows the average time per rendering pass (resolution dependent) single threaded on a Core $22.33 \mathrm{GHz}$. As each pass is independent, the computation scales well to multi-core machines. It can be seen that in scenes with many glossy or singular materials the number of average VPL per pass drops while the time spent for the eye paths automatically increases.

tracing. In Proceedings of Third International Conference on Computational Graphics and Visualization Techniques (1993), pp. 145-153.

[PH04] Pharr M., Humphreys G.: Physically Based Rendering: From Theory to Implementation. Elsevier, 2004.

[RSK06] RAAB M., SEIbert D., Keller A.: Unbiased Global Illumination with Participating Media. In Proc. Monte Carlo and Quasi-Monte Carlo Methods 2006. Springer, 2006, pp. 591-605.

[Sch94] SCHLICK C.: An inexpensive BRDF model for physically-based rendering. Computer Graphics Forum 13 (1994), 233-246.

[Shi06] SHIRLEY P.: State of the art in interactive ray tracing. In ACM SIGGRAPH 2006 Courses (2006).

[SIMP07] Segovia B., IEhl J.-C., Mitanchey R., PÉRoche B.: Metropolis Instant Radiosity. Computer Graphics Forum 26, 3 (2007), 425-434.

[SW00] SuYKens F., Willems Y.: Adaptive Filtering for Progressive Monte Carlo Image Rendering. In Eighth International Conference in Central Europe on Computer Graphics, Visualization and Interactive Digital Media (WSCG 2000) (Plzen, Czech Republic, 2000).

[Vea97] VEACH E.: Robust Monte Carlo Methods for Light Transport Simulation. PhD thesis, Stanford University, 1997.

[VG94] VEACH E., GuiBas L.: Bidirectional estimators for light transport. In Rendering Techniques '94 (Proc. of the Fifth Eurographics Workshop on Rendering) (1994), pp. 147 - 161.

[VG95] VEACH E., GuIBAS L.: Optimally compining sampling techniques for Monte Carlo rendering. ACM Transactions on Graphics (Proc. SIGGRAPH 1995) (1995), 419-428.

[VG97] VEACH E., GuiBAs L.: Metropolis light transport. ACM Transactions on Graphics (Proc. SIGGRAPH 1997) (1997), 6576.

[WABG06] Walter B., Arbree A., Bala K., GreenberG D.: Multidimensional lightcuts. In SIGGRAPH '06: ACM SIGGRAPH 2006 Papers (2006), pp. 1081-1088.

[War92] WARD G.: Measuring and modeling anisotropic reflection. SIGGRAPH Comput. Graph. 26, 2 (1992), 265-272.

[WFA*05] Walter B., Fernandez S., ArbreE A., Bala K., Donikian M., Greenberg D.: Lightcuts: a scalable approach to illumination. In SIGGRAPH '05: ACM SIGGRAPH 2005 Papers (2005), pp. 1098-1107. 


\section{Liste der bisher erschienenen Ulmer Informatik-Berichte}

Einige davon sind per FTP von ftp.informatik.uni-ulm. de erhältlich

Die mit * markierten Berichte sind vergriffen

List of technical reports published by the University of Ulm

Some of them are available by FTP from ftp. informatik. uni-ulm. de

Reports marked with * are out of print

91-01 Ker-I Ko, P. Orponen, U. Schöning, O. Watanabe

Instance Complexity

91-02* K. Gladitz, H. Fassbender, H. Vogler

Compiler-Based Implementation of Syntax-Directed Functional Programming

91-03* Alfons Geser

Relative Termination

91-04* J. Köbler, U. Schöning, J. Toran

Graph Isomorphism is low for PP

91-05 Johannes Köbler, Thomas Thierauf

Complexity Restricted Advice Functions

91-06* Uwe Schöning

Recent Highlights in Structural Complexity Theory

91-07* F. Green, J. Köbler, J. Toran

The Power of Middle Bit

91-08* V.Arvind, Y. Han, L. Hamachandra, J. Köbler, A. Lozano, M. Mundhenk, A. Ogiwara, U. Schöning, R. Silvestri, T. Thierauf

Reductions for Sets of Low Information Content

92-01* Vikraman Arvind, Johannes Köbler, Martin Mundhenk

On Bounded Truth-Table and Conjunctive Reductions to Sparse and Tally Sets

92-02* Thomas Noll, Heiko Vogler

Top-down Parsing with Simulataneous Evaluation of Noncircular Attribute Grammars

92-03 Fakultät für Informatik

17. Workshop über Komplexitätstheorie, effiziente Algorithmen und Datenstrukturen

92-04* V. Arvind, J. Köbler, M. Mundhenk

Lowness and the Complexity of Sparse and Tally Descriptions

92-05* Johannes Köbler

Locating P/poly Optimally in the Extended Low Hierarchy

92-06* Armin Kühnemann, Heiko Vogler

Synthesized and inherited functions -a new computational model for syntax-directed semantics

92-07* Heinz Fassbender, Heiko Vogler

A Universal Unification Algorithm Based on Unification-Driven Leftmost Outermost Narrowing 
92-08* Uwe Schöning

On Random Reductions from Sparse Sets to Tally Sets

92-09* $\quad$ Hermann von Hasseln, Laura Martignon

Consistency in Stochastic Network

92-10 Michael Schmitt

A Slightly Improved Upper Bound on the Size of Weights Sufficient to Represent Any Linearly Separable Boolean Function

92-11 Johannes Köbler, Seinosuke Toda

On the Power of Generalized MOD-Classes

92-12 V. Arvind, J. Köbler, M. Mundhenk

Reliable Reductions, High Sets and Low Sets

92-13 Alfons Geser

On a monotonic semantic path ordering

92-14* Joost Engelfriet, Heiko Vogler

The Translation Power of Top-Down Tree-To-Graph Transducers

93-01 Alfred Lupper, Konrad Froitzheim

AppleTalk Link Access Protocol basierend auf dem Abstract Personal

Communications Manager

93-02 M.H. Scholl, C. Laasch, C. Rich, H.-J. Schek, M. Tresch

The COCOON Object Model

93-03 Thomas Thierauf, Seinosuke Toda, Osamu Watanabe

On Sets Bounded Truth-Table Reducible to P-selective Sets

93-04 Jin-Yi Cai, Frederic Green, Thomas Thierauf

On the Correlation of Symmetric Functions

93-05 K.Kuhn, M.Reichert, M. Nathe, T. Beuter, C. Heinlein, P. Dadam

A Conceptual Approach to an Open Hospital Information System

93-06 Klaus Gaßner

Rechnerunterstützung für die konzeptuelle Modellierung

93-07 Ullrich Keßler, Peter Dadam

Towards Customizable, Flexible Storage Structures for Complex Objects

94-01 Michael Schmitt

On the Complexity of Consistency Problems for Neurons with Binary Weights

94-02 Armin Kühnemann, Heiko Vogler

A Pumping Lemma for Output Languages of Attributed Tree Transducers

94-03 Harry Buhrman, Jim Kadin, Thomas Thierauf

On Functions Computable with Nonadaptive Queries to NP

94-04 Heinz Faßbender, Heiko Vogler, Andrea Wedel

Implementation of a Deterministic Partial E-Unification Algorithm for Macro Tree

Transducers 
94-05 V. Arvind, J. Köbler, R. Schuler

On Helping and Interactive Proof Systems

94-06 Christian Kalus, Peter Dadam

Incorporating record subtyping into a relational data model

94-07 Markus Tresch, Marc H. Scholl

A Classification of Multi-Database Languages

94-08 Friedrich von Henke, Harald Rueß

Arbeitstreffen Typtheorie: Zusammenfassung der Beiträge

94-09 F.W. von Henke, A. Dold, H. Rueß, D. Schwier, M. Strecker

Construction and Deduction Methods for the Formal Development of Software

94-10 Axel Dold

Formalisierung schematischer Algorithmen

94-11 Johannes Köbler, Osamu Watanabe

New Collapse Consequences of NP Having Small Circuits

94-12 Rainer Schuler

On Average Polynomial Time

94-13 Rainer Schuler, Osamu Watanabe

Towards Average-Case Complexity Analysis of NP Optimization Problems

94-14 Wolfram Schulte, Ton Vullinghs

Linking Reactive Software to the X-Window System

94-15 Alfred Lupper

Namensverwaltung und Adressierung in Distributed Shared Memory-Systemen

94-16 Robert Regn

Verteilte Unix-Betriebssysteme

94-17 Helmuth Partsch

Again on Recognition and Parsing of Context-Free Grammars:

Two Exercises in Transformational Programming

94-18 Helmuth Partsch

Transformational Development of Data-Parallel Algorithms: an Example

95-01 Oleg Verbitsky

On the Largest Common Subgraph Problem

95-02 Uwe Schöning

Complexity of Presburger Arithmetic with Fixed Quantifier Dimension

95-03 Harry Buhrman,Thomas Thierauf

The Complexity of Generating and Checking Proofs of Membership

95-04 Rainer Schuler, Tomoyuki Yamakami

Structural Average Case Complexity

95-05 Klaus Achatz, Wolfram Schulte

Architecture Indepentent Massive Parallelization of Divide-And-Conquer Algorithms 
95-06 Christoph Karg, Rainer Schuler

Structure in Average Case Complexity

95-07 P. Dadam, K. Kuhn, M. Reichert, T. Beuter, M. Nathe

ADEPT: Ein integrierender Ansatz zur Entwicklung flexibler, zuverlässiger kooperierender Assistenzsysteme in klinischen Anwendungsumgebungen

95-08 Jürgen Kehrer, Peter Schulthess

Aufbereitung von gescannten Röntgenbildern zur filmlosen Diagnostik

95-09 Hans-Jörg Burtschick, Wolfgang Lindner

On Sets Turing Reducible to P-Selective Sets

95-10 Boris Hartmann

Berücksichtigung lokaler Randbedingung bei globaler Zieloptimierung mit neuronalen Netzen am Beispiel Truck Backer-Upper

95-12 Klaus Achatz, Wolfram Schulte

Massive Parallelization of Divide-and-Conquer Algorithms over Powerlists

95-13 Andrea Mößle, Heiko Vogler

Efficient Call-by-value Evaluation Strategy of Primitive Recursive Program Schemes

95-14 Axel Dold, Friedrich W. von Henke, Holger Pfeifer, Harald Rueß

A Generic Specification for Verifying Peephole Optimizations

96-01 Ercüment Canver, Jan-Tecker Gayen, Adam Moik

Formale Entwicklung der Steuerungssoftware für eine elektrisch ortsbediente Weiche mit VSE

96-02 Bernhard Nebel

Solving Hard Qualitative Temporal Reasoning Problems: Evaluating the Efficiency of Using the ORD-Horn Class

96-03 Ton Vullinghs, Wolfram Schulte, Thilo Schwinn

An Introduction to TkGofer

96-04 Thomas Beuter, Peter Dadam

Anwendungsspezifische Anforderungen an Workflow-Mangement-Systeme am

Beispiel der Domäne Concurrent-Engineering

96-05 Gerhard Schellhorn, Wolfgang Ahrendt

Verification of a Prolog Compiler - First Steps with KIV

96-06 Manindra Agrawal, Thomas Thierauf

Satisfiability Problems

96-07 Vikraman Arvind, Jacobo Torán

A nonadaptive NC Checker for Permutation Group Intersection

96-08 David Cyrluk, Oliver Möller, Harald Rueß

An Efficient Decision Procedure for a Theory of Fix-Sized Bitvectors with Composition and Extraction

96-09 Bernd Biechele, Dietmar Ernst, Frank Houdek, Joachim Schmid, Wolfram Schulte Erfahrungen bei der Modellierung eingebetteter Systeme mit verschiedenen SA/RTAnsätzen 
96-10 Falk Bartels, Axel Dold, Friedrich W. von Henke, Holger Pfeifer, Harald Rueß Formalizing Fixed-Point Theory in PVS

96-11 Axel Dold, Friedrich W. von Henke, Holger Pfeifer, Harald Rueß Mechanized Semantics of Simple Imperative Programming Constructs

96-12 Axel Dold, Friedrich W. von Henke, Holger Pfeifer, Harald Rueß Generic Compilation Schemes for Simple Programming Constructs

96-13 Klaus Achatz, Helmuth Partsch

From Descriptive Specifications to Operational ones: A Powerful Transformation Rule, its Applications and Variants

97-01 Jochen Messner

Pattern Matching in Trace Monoids

97-02 Wolfgang Lindner, Rainer Schuler

A Small Span Theorem within P

97-03 Thomas Bauer, Peter Dadam

A Distributed Execution Environment for Large-Scale Workflow Management Systems with Subnets and Server Migration

97-04 Christian Heinlein, Peter Dadam

Interaction Expressions - A Powerful Formalism for Describing Inter-Workflow Dependencies

97-05 Vikraman Arvind, Johannes Köbler

On Pseudorandomness and Resource-Bounded Measure

97-06 Gerhard Partsch

Punkt-zu-Punkt- und Mehrpunkt-basierende LAN-Integrationsstrategien für den digitalen Mobilfunkstandard DECT

97-07 Manfred Reichert, Peter Dadam

$A D E P T_{\text {flex }}$ - Supporting Dynamic Changes of Workflows Without Loosing Control

97-08 Hans Braxmeier, Dietmar Ernst, Andrea Mößle, Heiko Vogler

The Project NoName - A functional programming language with its development environment

97-09 Christian Heinlein

Grundlagen von Interaktionsausdrücken

97-10 Christian Heinlein

Graphische Repräsentation von Interaktionsausdrücken

97-11 Christian Heinlein

Sprachtheoretische Semantik von Interaktionsausdrücken

97-12 Gerhard Schellhorn, Wolfgang Reif

Proving Properties of Finite Enumerations: A Problem Set for Automated Theorem Provers 
97-13 Dietmar Ernst, Frank Houdek, Wolfram Schulte, Thilo Schwinn

Experimenteller Vergleich statischer und dynamischer Softwareprüfung für eingebettete Systeme

97-14 Wolfgang Reif, Gerhard Schellhorn

Theorem Proving in Large Theories

97-15 Thomas Wennekers

Asymptotik rekurrenter neuronaler Netze mit zufälligen Kopplungen

97-16 Peter Dadam, Klaus Kuhn, Manfred Reichert

Clinical Workflows - The Killer Application for Process-oriented Information Systems?

97-17 Mohammad Ali Livani, Jörg Kaiser

EDF Consensus on CAN Bus Access in Dynamic Real-Time Applications

97-18 Johannes Köbler,Rainer Schuler

Using Efficient Average-Case Algorithms to Collapse Worst-Case Complexity Classes

98-01 Daniela Damm, Lutz Claes, Friedrich W. von Henke, Alexander Seitz, Adelinde

Uhrmacher, Steffen Wolf

Ein fallbasiertes System für die Interpretation von Literatur zur Knochenheilung

98-02 Thomas Bauer, Peter Dadam

Architekturen für skalierbare Workflow-Management-Systeme - Klassifikation und Analyse

98-03 Marko Luther, Martin Strecker

A guided tour through Typelab

98-04 Heiko Neumann, Luiz Pessoa

Visual Filling-in and Surface Property Reconstruction

98-05 Ercüment Canver

Formal Verification of a Coordinated Atomic Action Based Design

98-06 Andreas Küchler

On the Correspondence between Neural Folding Architectures and Tree Automata

98-07 Heiko Neumann, Thorsten Hansen, Luiz Pessoa

Interaction of ON and OFF Pathways for Visual Contrast Measurement

98-08 Thomas Wennekers

Synfire Graphs: From Spike Patterns to Automata of Spiking Neurons

98-09 Thomas Bauer, Peter Dadam

Variable Migration von Workflows in ADEPT

98-10 Heiko Neumann, Wolfgang Sepp

Recurrent V1 - V2 Interaction in Early Visual Boundary Processing

98-11 Frank Houdek, Dietmar Ernst, Thilo Schwinn

Prüfen von C-Code und Statmate/Matlab-Spezifikationen: Ein Experiment 
98-12 Gerhard Schellhorn

Proving Properties of Directed Graphs: A Problem Set for Automated Theorem Provers

98-13 Gerhard Schellhorn, Wolfgang Reif

Theorems from Compiler Verification: A Problem Set for Automated Theorem

Provers

98-14 Mohammad Ali Livani

SHARE: A Transparent Mechanism for Reliable Broadcast Delivery in CAN

98-15 Mohammad Ali Livani, Jörg Kaiser

Predictable Atomic Multicast in the Controller Area Network (CAN)

99-01 Susanne Boll, Wolfgang Klas, Utz Westermann

A Comparison of Multimedia Document Models Concerning Advanced Requirements

99-02 Thomas Bauer, Peter Dadam

Verteilungsmodelle für Workflow-Management-Systeme - Klassifikation und

Simulation

99-03 Uwe Schöning

On the Complexity of Constraint Satisfaction

99-04 Ercument Canver

Model-Checking zur Analyse von Message Sequence Charts über Statecharts

99-05 Johannes Köbler, Wolfgang Lindner, Rainer Schuler

Derandomizing RP if Boolean Circuits are not Learnable

99-06 Utz Westermann, Wolfgang Klas

Architecture of a DataBlade Module for the Integrated Management of Multimedia Assets

99-07 Peter Dadam, Manfred Reichert

Enterprise-wide and Cross-enterprise Workflow Management: Concepts, Systems, Applications. Paderborn, Germany, October 6, 1999, GI-Workshop Proceedings, Informatik '99

99-08 Vikraman Arvind, Johannes Köbler

Graph Isomorphism is Low for $\mathrm{ZPP}^{\mathrm{NP}}$ and other Lowness results

99-09 Thomas Bauer, Peter Dadam

Efficient Distributed Workflow Management Based on Variable Server Assignments

2000-02 Thomas Bauer, Peter Dadam

Variable Serverzuordnungen und komplexe Bearbeiterzuordnungen im Workflow-

Management-System ADEPT

2000-03 Gregory Baratoff, Christian Toepfer, Heiko Neumann

Combined space-variant maps for optical flow based navigation

2000-04 Wolfgang Gehring

Ein Rahmenwerk zur Einführung von Leistungspunktsystemen 
2000-05 Susanne Boll, Christian Heinlein, Wolfgang Klas, Jochen Wandel

Intelligent Prefetching and Buffering for Interactive Streaming of MPEG Videos

2000-06 Wolfgang Reif, Gerhard Schellhorn, Andreas Thums

Fehlersuche in Formalen Spezifikationen

2000-07 Gerhard Schellhorn, Wolfgang Reif (eds.)

FM-Tools 2000: The $4^{\text {th }}$ Workshop on Tools for System Design and Verification

2000-08 Thomas Bauer, Manfred Reichert, Peter Dadam

Effiziente Durchführung von Prozessmigrationen in verteilten Workflow-

Management-Systemen

2000-09 Thomas Bauer, Peter Dadam

Vermeidung von Überlastsituationen durch Replikation von Workflow-Servern in ADEPT

2000-10 Thomas Bauer, Manfred Reichert, Peter Dadam

Adaptives und verteiltes Workflow-Management

2000-11 Christian Heinlein

Workflow and Process Synchronization with Interaction Expressions and Graphs

2001-01 Hubert Hug, Rainer Schuler

DNA-based parallel computation of simple arithmetic

2001-02 Friedhelm Schwenker, Hans A. Kestler, Günther Palm

3-D Visual Object Classification with Hierarchical Radial Basis Function Networks

2001-03 Hans A. Kestler, Friedhelm Schwenker, Günther Palm

RBF network classification of ECGs as a potential marker for sudden cardiac death

2001-04 Christian Dietrich, Friedhelm Schwenker, Klaus Riede, Günther Palm

Classification of Bioacoustic Time Series Utilizing Pulse Detection, Time and

Frequency Features and Data Fusion

2002-01 Stefanie Rinderle, Manfred Reichert, Peter Dadam

Effiziente Verträglichkeitsprüfung und automatische Migration von Workflow-

Instanzen bei der Evolution von Workflow-Schemata

2002-02 Walter Guttmann

Deriving an Applicative Heapsort Algorithm

2002-03 Axel Dold, Friedrich W. von Henke, Vincent Vialard, Wolfgang Goerigk

A Mechanically Verified Compiling Specification for a Realistic Compiler

2003-01 Manfred Reichert, Stefanie Rinderle, Peter Dadam

A Formal Framework for Workflow Type and Instance Changes Under Correctness Checks

2003-02 Stefanie Rinderle, Manfred Reichert, Peter Dadam

Supporting Workflow Schema Evolution By Efficient Compliance Checks

2003-03 Christian Heinlein

Safely Extending Procedure Types to Allow Nested Procedures as Values 
2003-04 Stefanie Rinderle, Manfred Reichert, Peter Dadam

On Dealing With Semantically Conflicting Business Process Changes.

2003-05 Christian Heinlein

Dynamic Class Methods in Java

2003-06 Christian Heinlein

Vertical, Horizontal, and Behavioural Extensibility of Software Systems

2003-07 Christian Heinlein

Safely Extending Procedure Types to Allow Nested Procedures as Values

(Corrected Version)

2003-08 Changling Liu, Jörg Kaiser

Survey of Mobile Ad Hoc Network Routing Protocols)

2004-01 Thom Frühwirth, Marc Meister (eds.)

First Workshop on Constraint Handling Rules

2004-02 Christian Heinlein

Concept and Implementation of $\mathrm{C}+++^{+}$, an Extension of $\mathrm{C}++^{+}$to Support User-Defined

Operator Symbols and Control Structures

2004-03 Susanne Biundo, Thom Frühwirth, Günther Palm(eds.)

Poster Proceedings of the 27th Annual German Conference on Artificial Intelligence

2005-01 Armin Wolf, Thom Frühwirth, Marc Meister (eds.)

19th Workshop on (Constraint) Logic Programming

2005-02 Wolfgang Lindner (Hg.), Universität Ulm, Christopher Wolf (Hg.) KU Leuven

2. Krypto-Tag - Workshop über Kryptographie, Universität Ulm

2005-03 Walter Guttmann, Markus Maucher

Constrained Ordering

2006-01 Stefan Sarstedt

Model-Driven Development with ACTIVECHARTS, Tutorial

2006-02 Alexander Raschke, Ramin Tavakoli Kolagari

Ein experimenteller Vergleich zwischen einer plan-getriebenen und einer

leichtgewichtigen Entwicklungsmethode zur Spezifikation von eingebetteten

Systemen

2006-03 Jens Kohlmeyer, Alexander Raschke, Ramin Tavakoli Kolagari

Eine qualitative Untersuchung zur Produktlinien-Integration über

Organisationsgrenzen hinweg

2006-04 Thorsten Liebig

Reasoning with OWL - System Support and Insights -

2008-01 H.A. Kestler, J. Messner, A. Müller, R. Schuler

On the complexity of intersecting multiple circles for graphical display 
2008-02 Manfred Reichert, Peter Dadam, Martin Jurisch,l Ulrich Kreher, Kevin Göser, Markus Lauer

Architectural Design of Flexible Process Management Technology

2008-03 Frank Raiser

Semi-Automatic Generation of CHR Solvers from Global Constraint Automata

2008-04 Ramin Tavakoli Kolagari, Alexander Raschke, Matthias Schneiderhan, Ian Alexander Entscheidungsdokumentation bei der Entwicklung innovativer Systeme für produktlinien-basierte Entwicklungsprozesse

2008-05 Markus Kalb, Claudia Dittrich, Peter Dadam

Support of Relationships Among Moving Objects on Networks

2008-06 Matthias Frank, Frank Kargl, Burkhard Stiller (Hg.)

WMAN 2008 - KuVS Fachgespräch über Mobile Ad-hoc Netzwerke

2008-07 M. Maucher, U. Schöning, H.A. Kestler

An empirical assessment of local and population based search methods with different degrees of pseudorandomness

2008-08 Henning Wunderlich

Covers have structure

2008-09 Karl-Heinz Niggl, Henning Wunderlich

Implicit characterization of FPTIME and NC revisited

2008-10 Henning Wunderlich

On span- $\mathrm{P}^{\mathrm{cc}}$ and related classes in structural communication complexity

2008-11 M. Maucher, U. Schöning, H.A. Kestler

On the different notions of pseudorandomness

2008-12 Henning Wunderlich

On Toda's Theorem in structural communication complexity

2008-13 Manfred Reichert, Peter Dadam

Realizing Adaptive Process-aware Information Systems with ADEPT2

2009-01 Peter Dadam, Manfred Reichert

The ADEPT Project: A Decade of Research and Development for Robust and Fexible Process Support

Challenges and Achievements

2009-02 Peter Dadam, Manfred Reichert, Stefanie Rinderle-Ma, Kevin Göser, Ulrich Kreher, Martin Jurisch

Von ADEPT zur AristaFlow ${ }^{\circledR}$ BPM Suite - Eine Vision wird Realität “Correctness by Construction” und flexible, robuste Ausführung von Unternehmensprozessen 
2009-03 Alena Hallerbach, Thomas Bauer, Manfred Reichert

Correct Configuration of Process Variants in Provop

2009-04 Martin Bader

On Reversal and Transposition Medians

2009-05 Barbara Weber, Andreas Lanz, Manfred Reichert

Time Patterns for Process-aware Information Systems: A Pattern-based Analysis

2009-06 Stefanie Rinderle-Ma, Manfred Reichert

Adjustment Strategies for Non-Compliant Process Instances

2009-07 H.A. Kestler, B. Lausen, H. Binder H.-P. Klenk. F. Leisch, M. Schmid

Statistical Computing 2009 - Abstracts der 41. Arbeitstagung

2009-08 Ulrich Kreher, Manfred Reichert, Stefanie Rinderle-Ma, Peter Dadam

Effiziente Repräsentation von Vorlagen- und Instanzdaten in Prozess-ManagementSystemen

2009-09 Dammertz, Holger, Alexander Keller, Hendrik P.A. Lensch

Progressive Point-Light-Based Global Illumination 
Ulmer Informatik-Berichte

ISSN 0939-5091

Herausgeber:

Universität UIm

Fakultät für Ingenieurwissenschaften und Informatik 89069 Ulm 